\title{
Topical Immunotherapy of Alopecia Areata: A Large Retrospective Study
}

\author{
Nicola Zerbinati (D) C Cristina Esposito - Edoardo D'Este • \\ Alberto Calligaro · Rossano Valsecchi
}

Received: November 30, 2017 / Published online: February 13, 2018

(C) The Author(s) 2018. This article is an open access publication

\begin{abstract}
Introduction: Topical immunotherapy is frequently used in the treatment of alopecia areata (AA) although few studies report long-term follow-up. Our goals were to determine the efficacy and the prognostic factors of topical immunotherapy in a large cohort of patients with AA treated in the departments of Dermatology and Venereology of Bergamo, Como and Pavia, from 1978 to January 2016.
\end{abstract}

Methods: A total of 252 patients with AA were evaluated retrospectively.

Results: All our patients developed an allergic reaction to a $2 \%$ solution of dinitrochlorobenzene (DNCB) or squaric acid dibutylester (SADBE) or diphenylcyclopropenone (DPCP). No patients discontinued therapy because of

Enhanced content To view enhanced content for this article go to https://doi.org/10.6084/m9.figshare. 5831613.

\section{N. Zerbinati $(\bowtie)$}

Department of Surgical and Morphological Sciences, University of Insubria (Varese), Varese, Italy

e-mail: nzerbinati@centro-medico.it

C. Esposito - E. D'Este · R. Valsecchi Dermatology Department of Centro Medico Polispecialistico, Pavia, Italy

\section{A. Calligaro}

Department of Public Health, Experimental and Forensic Medicine, Unit of Histology and Embryology, University of Pavia, Pavia, Italy side effects. In total 112 patients (44.05\%, $p<0.001$ ) had a good response. Statistical analysis revealed that the main prognostic factors influencing the clinical results were the severity of hair loss at the beginning of therapy, the duration of AA, a history of atopy (particularly eczema), and the early development of sensitization to the three sensitizers.

Conclusion: Topical immunotherapy in patients with AA is quite effective, mostly well tolerated, and provides prolonged therapeutic benefits.

Keywords: Alopecia areata; Hair loss; Immunotherapy

\section{INTRODUCTION}

Alopecia areata (AA) is a skin disorder characterized by patchy, confluent, or diffuse hair loss which results in hair loss on the scalp and other areas of the body to various extents. AA is subdivided into localized alopecia areata (AL), alopecia areata totalis (AT), and alopecia areata universalis (AU), reflecting respectively patchy, full-scalp, and full-body hair loss [1]. The prevalence of AA is between $0.1 \%$ and $0.2 \%$ [2] with a calculated lifetime risk of $2 \%$ and most commonly the onset is before the age of 40 years [3]. AA is related to various atopic and autoimmune diseases [4], and in affected people there is a high prevalence of eczema, asthma, 
nail changes, thyroid autoimmunity, vitamin D deficiency, vitiligo, and psoriasis [5]. Despite being considered a benign pathology, patients suffering from AA have poor health-related quality of life scores (HRQOL) - the higher the extent, the lower the score-and, in addition, a high incidence of psychiatric morbidity, especially anxiety and mood disturbance [6].

The pathogenesis of AA is still incompletely understood. The principal areas under investigation are genetic constitution, non-specific immune reactions, and organ-specific autoimmune reactions. Genetic factors can play an important role, as proven by the fact that monozygotic twins can have similar times of onset and patterns of hair loss [7] and, in addition, some patients have a strong family history [8]; these data suggest that AA can be inherited. Some HLA regions, on human chromosome 6 , are associated with genetic susceptibility to AA. A genome-wide association study was conducted on extended human families with multiple AA patients and identified some intervals on human chromosomes $6,10,16$, and 18 both inside and outside the HLA gene cluster as potential AA susceptibility loci [9].

Many potential environmental stimuli such as hormonal fluctuation, infectious agents, and vaccinations may also contribute to determine onset, hair loss pattern, and severity of disease [10]. The theory currently most accredited is that there is an interaction between genetic and environmental factors that triggers the disease. Several hypotheses have been suggested to explain the development of autoimmune disease, the most reasonable of which focuses on hair follicle immune privilege collapse or on the inappropriate presentation of antigens to the immune system during normal hair follicle cycling [11].

Up to $50 \%$ of patients experience spontaneous hair regrowth while many subjects suffer from a chronic disease that is a challenge for dermatologists to treat [12].

In 2012, the British Association of Dermatologists updated the 2003 guidelines for the management of AA [13]. For children, topical mid-potent corticosteroids are the first-line therapy. For adult patients with less than 50\% scalp involvement intralesional corticosteroids can be taken into consideration, while for adult patients with more than 50\% scalp involvement topical immunotherapy is the first-line therapy [14].

The effectiveness of topical immunotherapies has already been demonstrated in several well-controlled studies: in 2010 a meta-analysis concluded that topical immunotherapies are effective in up to $50-60 \%$ of cases, with a relapse rate of $62 \%$ with median time to relapse of 2.5 years [15].

In this study, we retrospectively evaluated efficacy and safety of topical immunotherapy in 252 patients with AA. The purpose of the study was also to identify possible prognostic factors that could modify the clinical outcome of the therapy and to review critically the standardized protocol of application.

\section{METHODS}

In this retrospective study we enrolled 252 subjects affected by AA with a mean age of 35.5 years (range 5-61 years old) that were treated with topical immunotherapy from 1978 to 2016 in the Department of Dermatology and Venerology of Bergamo, Como and Pavia.

The exclusion criteria were history of cardiovascular disease, pregnancy/lactation, and treatment with steroids per os for any reason. The inclusion criteria were diagnosis of AA and no previous treatment with topical immunotherapy without success.

All patients underwent a standardized diagnostic protocol with pull test and video-dermoscopy that showed the typical aspect of the illness with yellow dots and exclamation hair, in patients with localized AA; in cases of borderline diagnosis, in patients with localized AA of recent onset, histological examination was performed to identify the typical pattern of the disease with a peribulbar "swarm of bees" lymphocytic infiltrate composed of CD4 and CD8. The extent of AA at the first visit was evaluated in accordance with National Alopecia Areata Foundation guidelines.

The demographic and clinical data are shown in Table 1 . Nine patients had a family history of vitiligo and, interestingly, the same 
Table 1 Demographic and clinical data

\begin{tabular}{lc}
\hline Demographic data & 252 \\
\hline Number of patients & 35.5 \\
Age at beginning of therapy (years) \\
Mean & $5-61$ \\
Range & \\
\hline
\end{tabular}

\begin{tabular}{lrl}
\hline Clinical data & No. & $\%$ \\
\hline Type of alopecia areata & & \\
AU & 44 & 17.46 \\
AT & 68 & 26.98 \\
AL & 140 & 55.56 \\
$S>30 \%$ & 47 & 18.65 \\
$S>40 \%$ & 31 & 12.30 \\
$S>50 \%$ & 62 & 24.60
\end{tabular}

Comorbidities

Personal history

$\begin{array}{lrr}\text { Atopy } & 23 & 9.13 \\ \text { Thyroid dysfunction } & 14 & 5.56 \\ \text { Vitiligo } & 9 & 3.57 \\ \text { Family history } & & \\ \text { Atopy } & 14 & 5.56 \\ \text { Thyroid dysfunction } & 23 & 9.13 \\ \text { Vitiligo } & 9 & 3.57 \\ \text { Positive family history of AA } & 34 & 13.49\end{array}$

$A U$ alopecia universalis, $A T$ alopecia totalis, $A L$ alopecia localized, $S$ scalp involvement, $A A$ alopecia areata

number of patients had vitiligo themselves. Fourteen patients had a family history of atopy; on the other hand, 23 patients, had a personal history of eczema, rhinitis, and asthma. A total of 23 patients had a positive family history of thyroid disease, while 14 had a positive personal history of thyroid disease. Thirty-four patients had a family history of AA.

Contact sensitizers used included dinitrochlorobenzene (DNCB), squaric acid dibutylester (SADBE), and diphenylcyclopropenone (DPCP).

We started our experience in 1978 using $\mathrm{DNCB}$, a substance which is not generally used today because it has been shown to be mutagenic in the Ames test and genotoxic by sister chromatid exchange in human skin fibroblast [16]. In 1981, we introduced SADBE that is not mutagenic but is scarcely stable in acetone. Some years later, in 1990, we started to use DPCP, a nonmutagenic sensitizer, which is less expensive and more stable in acetone than DNCB is. This sensitizer is able to induce the expression of immunoregulatory molecules that are important in the pathogenesis of AA in healthy skin [17].

Topical immunotherapy was performed following a standard protocol of application: the most severely affected site of the scalp was chosen for the initial treatment; a $2 \%$ solution of DNCB or SADBE or DPCP, in acetone, was applied onto a $4 \mathrm{~cm}^{2}$ circular area. After 2 days, this solution was removed by washing. Two weeks after sensitization, a $0.001 \%$ solution of the selected sensitizer was applied to the same half of the scalp in order to rule out spontaneous regrowth that would have appeared on the untreated areas as well. The concentration of sensitizer was increased gradually each week until a mild dermatitis reaction with low grade erythema and mild pruritus for 2 days was obtained. All 252 patients were successfully sensitized and no patients discontinued immunotherapy because of side effects.

The concentration of the contact sensitizer was adjusted to the patient's reactivity against the allergens and when the appropriate concentration had been found the sensitizer was applied weekly to the sensitized area. Subsequently, when an initial regrowth occurred in the treated area (on average after 12-18 weeks) the solution was applied to the whole scalp. Once a hair regrowth had been achieved, to avoid relapse, topical immunotherapy was tapered off by prolonging the application intervals to 2 weeks, then to 3 weeks, after to 4 weeks, and finally discontinued.

In our study treatment success was defined as at least $50 \%$ of terminal hair regrowth. The patient's hair regrowth was evaluated every 
6 months for 4 years, when applicable. If there was no improvement after 12 months of treatment, the patient was considered to be a nonresponder and the therapy was interrupted. Full information regarding the therapy, including expected clinical outcomes and possible side effects, were given to the patient and a signed informed consent was obtained before treatment.

Statistical analysis was performed using the $\mathrm{R}$ integrated suite by the R Project software (version 3.3.3), with software package exact $2 \times 2$ (version 1.5.2). Statistical significance was assessed using the exact test, and the results were considered significant for $p<0.05$.

All procedures performed in studies involving human participants were in accordance with the ethical standards of the institutional and/or national research committee and with the 1964 Declaration of Helsinki and its later amendments or comparable ethical standards. Informed consent was obtained from all individual participants included in the study.

\section{RESULTS}

A good response to the therapy was achieved in 112 patients (44.05\%); hair regrowth was achieved in $50.98 \%$ of patients treated with DNCB, $45.71 \%$ of patients treated with SADBE, and $34.43 \%$ of patients treated with DPCP.

According to the extension of AA we divided our cohort of patients into three groups:

1. We observed 140 patients with localized AA (S) that were classified into three additional subgroups:

(a) 47 patients with $S>30 \%$ : 12 patients were treated with DNCB among which ten had hair regrowth, 29 patients were treated with SADBE among which 15 had hair regrowth, six patients were treated with DPCP and no one had hair regrowth.

Conclusion 47 patients, 25 (53.19\%) had hair regrowth.

(b) 31 patients with $S>40 \%$ : five patients were treated with DNCB and five of them had hair regrowth, 16 patients were treated with SADBE and ten of them had hair regrowth, ten patients were treated with DPCP and four of them had hair regrowth.

Conclusion 31 patients, 18 (58.06\%) had hair regrowth.

(c) 62 patients with $S>50 \%$ : ten patients were treated with DNCB and we observed seven cases of hair regrowth, 40 patients were treated with SADBE among whom we have registered 22 cases of hair regrowth, 12 patients were treated with DPCP and five of them had a good response to the therapy.

Conclusion 62 patients, 34 (54.84\%) had hair regrowth.

2. We observed 68 patients with AT; in this group, ten patients were treated with DNCB and we observed hair regrowth in three cases, 38 patients were treated with SADBE and hair growth was obtained in 13 subjects, 20 patients were treated with DPCP and ten of them had hair growth.

Conclusion 68 patients, 26 (38.24\%) had hair regrowth.

3. We observed 44 patients with $\mathrm{AU}$; in this group, 14 patients were treated with DNCB and two had hair regrowth, 17 patients were treated with SADBE and four of them had hair regrowth, 13 patients were treated with DPCP and we observed a partial regrowth in two cases.

Conclusion 44 patients: 8 (18.18\%) had hair regrowth.

Overall we treated 51 patients with DNCB, 140 patients with SADBE, and 61 patients with DPCP.

Similar results were observed with each contact sensitizer (DNCB, SADBE, and DPCP) with comparable main time of relapse; in our long-term follow-up study the therapeutic results obtained were essentially the same, irrespective of the type of sensitizer used.

The patients who experienced hair regrowth had a follow-up of 48 months, with a visit every 6 months when applicable, and none had recurrence of the disease. In general, during the long time of topical immunotherapy (more than 35 years) we observed that $46 \%$ of patients had a relapse during the therapy and were treated again. 
During the first few years of our experience with topical immunotherapy we attempted to determine some criteria that might influence the clinical results, but we did not obtain relevant conclusions.

At the end of our experience, the duration of AA, a history of atopy (particularly eczema), and the severity and early development of flare-up (skin reaction at the site of the first sensitizing dose application of topical allergens) were the main factors that had influenced the clinical results.

From the clinical point of view, we observed that the significant prognostic factors influencing positive outcomes were the severity of flareup and the maintenance of a low-grade contact eczema.

The success rate with relative confidence interval at 95\%, $p$ value, and the odds success rate between the sensitizers standardized by each single group of AA are reported in Tables 2 and 3.

\section{DISCUSSION}

The primary aim of our study was to assess the validity of topical immunotherapy in a population of 252 patients with AA.

The main result was satisfactory with $44.05 \%$ of patients experiencing at least $50 \%$ of terminal hair regrowth.

The evidence-based results of topical immunotherapy confirm the theory that AA is related to an autoimmune etiology. The mechanism that is operative in this therapy is mainly due to local immunomodulation through two components: the synthetic antigen acts in a competitive way with the antigen involved in pathogenesis of AA and consequently represents an alternative target for the immune system. Moreover, because of a prolonged immune stimulation in treated patients, we can observe an increase of Treg lymphocytes which contrast the immune reaction towards the follicle $[17,18]$.

The average of success rates coming out of our data are in line with those reported in other studies in the literature. A 2010 meta-analysis, reported a mean efficacy of topical
Table 2 Success rate with relative confidence interval at $95 \%$ and $p$ value in each group of patients

\begin{tabular}{|c|c|c|}
\hline & \% Success (95\% CI) & $p$ value \\
\hline \multicolumn{3}{|c|}{ Patients with AU $(n=44)$} \\
\hline Total & $18.18(8.19 ; 32.71)$ & $<0.001$ \\
\hline Treated with DNCB & $14.29(1.78 ; 42.81)$ & $<0.001$ \\
\hline Treated with SADBE & $23.53(6.81 ; 49.9)$ & $<0.001$ \\
\hline Treated with DPCP & $15.38(1.92 ; 45.45)$ & $<0.001$ \\
\hline \multicolumn{3}{|c|}{ Patients with AT $(n=68)$} \\
\hline Total & $38.24(26.71 ; 50.82)$ & $<0.001$ \\
\hline Treated with DNCB & $30.00(6.67 ; 65.25)$ & $<0.001$ \\
\hline Treated with SADBE & $34.21(19.63 ; 51.35)$ & $<0.001$ \\
\hline Treated with DPCP & $50.00(27.20 ; 72.80)$ & $<0.001$ \\
\hline \multicolumn{3}{|c|}{ Patients with AL $(n=140)$} \\
\hline Total & $55.00(46.37 ; 63.41)$ & $<0.001$ \\
\hline Treated with DNCB & 77.78 (57.74; 91.38) & $<0.001$ \\
\hline Treated with SADBE & $55.29(44.11 ; 66.09)$ & $<0.001$ \\
\hline Treated with DPCP & $32.14(15.88 ; 52.35)$ & $<0.001$ \\
\hline \multicolumn{3}{|c|}{ Patients with $S>30(n=47)$} \\
\hline Total & $53.19(38.08 ; 67.89)$ & $<0.001$ \\
\hline Treated with DNCB & $83.33(51.59 ; 97.91)$ & $<0.001$ \\
\hline Treated with SADBE & $51.72(32.53 ; 70.55)$ & $<0.001$ \\
\hline Treated with DPCP & $0.00(0.00 ; 45.93)$ & 1.000 \\
\hline \multicolumn{3}{|c|}{ Patients with $S>40(n=31)$} \\
\hline Total & $58.06(39.08 ; 75.45)$ & $<0.001$ \\
\hline Treated with DNCB & $80.00(28.36 ; 99.49)$ & $<0.001$ \\
\hline Treated with SADBE & $62.5(35.43 ; 84.80)$ & $<0.001$ \\
\hline Treated with DPCP & $40.00(12.16 ; 73.76)$ & $<0.001$ \\
\hline \multicolumn{3}{|c|}{ Patients with $S>50(n=62)$} \\
\hline Total & $54.84(41.68 ; 67.52)$ & $<0.001$ \\
\hline Treated with DNCB & $70.00(34.75 ; 93.33)$ & $<0.001$ \\
\hline Treated with SADBE & $55.00(38.49 ; 70.74)$ & $<0.001$ \\
\hline Treated with DPCP & $41.67(15.17 ; 72.33)$ & $<0.001$ \\
\hline \multicolumn{3}{|l|}{ Total patients $(n=252)$} \\
\hline Total & $44.05(37.82 ; 50.41)$ & $<0.001$ \\
\hline
\end{tabular}


Table 2 continued

\begin{tabular}{lcr}
\hline & \% Success (95\% CI) & $\boldsymbol{p}$ value \\
\hline Treated with DNCB & $50.98(36.60 ; 65.25)$ & $<0.001$ \\
Treated with SADBE & $45.71(37.28 ; 54.34)$ & $<0.001$ \\
Treated with DPCP & $34.43(22.73 ; 47.69)$ & $<0.001$ \\
\hline$C I$ confidence interval, $A U$ alopecia universalis, $A T$ \\
$\begin{array}{lll}\text { alopecia totalis, } A L \text { alopecia localized, DNCB } & \text { 2,4-dini- } \\
\text { trochlorobenzene, } S A D B E \text { squaric acid dibutylester, } D P C P\end{array}$ \\
$\begin{array}{l}\text { diphenylcyclopropenone, } S \text { scalp involvement } \\
\end{array}$
\end{tabular}

immunotherapy in up to $50-60 \%$ of patients with a range from $9 \%$ to $87 \%$ [15]. The great variability of success rates, even using the same standard protocol, can be explained by different experimental designs, inclusion and exclusion criteria, and duration of treatment in the single study.

In our population, no statistically significant difference $(p>0.05)$ was observed in success rates between different sensitizers: $50.98 \%$ of patients responded to DNCB, $45.71 \%$ responded to SADBE, and $34.43 \%$ responded to DPCP. These data can be explained because the sensitizing agents share the same mechanism of action: they all trigger a local allergic reaction that induces regression of the active phase of the disease and the progressive disappearance of alopecia patches.

The only exceptions are patients with AL with more than $30 \%$ involvement of the scalp. In this subpopulation, the sensitizer that seems to work best is DNCB with a success rate of $83.33 \%(p<0.001)$; however, this agent today is almost disused because it was found to be mutagenic with the Ames genotoxicity test since it leads to an increase in the exchange of chromosomal material between the sister chromatids in human skin fibroblasts. On the contrary, in this subpopulation, the sensitizer with the worst outcomes was DPCP, since not one of the patients had hair regrowth. This is in contrast to what is reported in the literature and is likely to be attributed, rather than to a real ineffectiveness of this immunosensitizer, to the individual variables related to the small number of subjects in this group (6 patients). In this group SADBE has shown an intermediate result with a response rate of $51.72 \%$ and, despite there being no statistically significant difference in success rate between DNCB and SADBE, the $p$ value is "borderline", i.e., close to 0.05 .

Some evidence on the similar efficacy of DPCP and SADBE was provided by Ohlmeier et al. who initially treated 142 patients with DPCP; after 6 months of therapy "non-responder" patients were treated using SADBE as sensitizer with the same standard protocol of application of therapy and, even changing the sensitizing agent, immunotherapy was not effective in this group of patients [19].

The secondary goal of our study was to define some possible prognostic factors that may affect the treatments success. During our experience, the most important independent prognostic factors on clinical outcomes were:

1. The extension of scalp involvement.

2. The duration of the disease and the duration of the current acute attack of AA before the beginning of the therapy.

3. The presence of atopy, mainly of eczema.

4. The early development of allergic contact dermatitis to sensitizers.

In our cohort, we registered better clinical results in patients with $\mathrm{AL}$, in all cases with a short time between the onset of alopecia and the beginning of the therapy, and in the people who developed allergic dermatitis early and with low concentrations of the sensitizing agent.

The statistical analysis of our data revealed a close correlation between the extension of AA before the therapy and the clinical outcome. The success rate in patients with AL was statistically significantly higher than in patients with AT or AU. At the same time, the average success rate in patients with AT was statistically significantly higher than in patients with AU. The same comparison made between AL subclasses has not shown statistically significant differences in treatment response. These data are in line with the literature; in fact several studies have demonstrated how the extension of AA is one of the main clinical prognostic factors. Ohlmeier et al. identified the severity of hair loss extension as the only prognostic factor, whereas Weise et al.'s population found the worst response to the immunotherapy in 
Table 3 Odds success rate between the sensitizers with relative confidence interval at $95 \%$ and $p$ value in each group of patients

\begin{tabular}{|c|c|c|}
\hline & Odd \% success $(95 \% \mathrm{CI})$ & $p$ value \\
\hline \multicolumn{3}{|l|}{ Patients with AU $(n=44)$} \\
\hline$\%$ Success SADBE - \% success DNCB & $9.24(-20.52 ; 36.02)$ & 0.909 \\
\hline \% Success DPCP - \% success DNCB & $1.10(-27.30 ; 30.28)$ & 0.938 \\
\hline$\%$ Success DPCP - \% success SADBE & $-8.14(-35.41 ; 22.82)$ & 0.909 \\
\hline \multicolumn{3}{|l|}{ Patients with AT $(n=68)$} \\
\hline \% Success SADBE - \% success DNCB & $4.21(-30.55 ; 31.62)$ & 0.827 \\
\hline \% Success DPCP - \% success DNCB & $20.00(-18.43 ; 50.99)$ & 0.475 \\
\hline$\%$ Success DPCP - \% success SADBE & $15.79(-10.80 ; 41.10)$ & 0.475 \\
\hline \multicolumn{3}{|l|}{ Patients with $\mathrm{AL}(n=140)$} \\
\hline$\%$ Success SADBE - \% success DNCB & $-22.48(-39.22 ;-1.47)$ & 0.036 \\
\hline$\%$ Success DPCP - \% success DNCB & $-45.63(-65.75 ;-19.47)$ & 0.002 \\
\hline$\%$ Success DPCP - \% success SADBE & $-23.15(-41.62 ;-1.62)$ & 0.036 \\
\hline \multicolumn{3}{|l|}{ Patients with $S>30(n=47)$} \\
\hline \% Success SADBE - \% success DNCB & $-31.61(-54.78 ; 1.66)$ & 0.062 \\
\hline \% Success DPCP - \% success DNCB & $-83.33(-95.98 ;-35.41)$ & 0.003 \\
\hline$\%$ Success DPCP - \% success SADBE & $-51.72(-67.29 ;-8.99)$ & 0.030 \\
\hline \multicolumn{3}{|l|}{ Patients with $S>40(n=31)$} \\
\hline$\%$ Success SADBE - \% success DNCB & $-17.50(-50.45 ; 33.47)$ & 0.517 \\
\hline \% Success DPCP - \% success DNCB & $-40.00(-74.32 ; 16.10)$ & 0.423 \\
\hline$\%$ Success DPCP - \% success SADBE & $-22.50(-56.22 ; 17.35)$ & 0.423 \\
\hline \multicolumn{3}{|l|}{ Patients with $S>50(n=42)$} \\
\hline$\%$ Success SADBE - \% success DNCB & $-15.00(-42.15 ; 20.25)$ & 0.433 \\
\hline \% Success DPC - \% success DNCB & $-28.33(-62.04 ; 14.43)$ & 0.433 \\
\hline$\%$ Success DPC - \% success SADBE & $-13.33(-42.20 ; 18.87)$ & 0.433 \\
\hline \multicolumn{3}{|l|}{ Total patients $(n=252)$} \\
\hline$\%$ Success SADBE - \% success DNCB & $-5.27(-21.02 ; 10.67)$ & 0.522 \\
\hline \% Success DPCP - \% success DNCB & $-16.55(-34.07 ; 1.93)$ & 0.207 \\
\hline \% Success DPCP - \% success SADBE & $-11.29(-25.18 ; 3.63)$ & 0.207 \\
\hline
\end{tabular}

$C I$ confidence interval, $A U$ alopecia universalis, $A T$ alopecia totalis, $A L$ alopecia localized, $D N C B$ 2,4-dinitrochlorobenzene, $S A D B E$ squaric acid dibutylester, $D P C P$ diphenylcyclopropenone, $S$ scalp involvement 
patients with AT or AU $[19,20]$ rather than in patients with AL. However, discordant data came from Gordon et al. and Firooz et al. who did not found any correlation between the extension of the hair loss and the response to treatment [21, 22].

As in our cohort of patients, other studies found a correlation between duration of AA and response to treatment $[20,21,23]$, while other authors did not come to the same conclusions $[22,24]$.

Another important prognostic factor in our analysis was the presence of atopy. Allergic patients had worse outcomes; however, the role of atopy as a prognostic factor is still highly debated. Results similar to our data have been described by several authors [20,21, 23], but on the other hand, some works showed the opposite results [19, 22, 24].

Other parameters that did not influence clinical outcomes in our investigation, unlike what we expected from literature data, were:

1. Sex

2. Age

3. Age of onset

4. Overuse of topical immunotherapy

5. Concomitant onychopathy.

In our population, 34 out of 252 patients (13.49\%) had a positive family history of AA; this evidence supports the hypothesis that the disease has a genetic component. Moreover, some authors also reported that a positive family history is associated with a worse prognosis with poor response to therapy [25] but, among the patients examined in our work, we did not found this correlation.

The major weakness of this study is the lack of a control group: this could have allowed the observers to conclude that any changes in the "active treatment group" were due to the treatment studied rather than to other factors.

One other limitation of the study is the relatively small numbers of subjects in the group with AL that represents the most common type of AA (140 patients; $55 \%$ of the total population).

Furthermore, in the subgroup of patients with $\mathrm{AL}$ and more than $30 \%$ scalp involvement, only six patients were treated with DPCP, making the analysis of the data in this subpopulation not really statistically significant, because of the individual variables related to the small number of subjects in the group.

\section{CONCLUSIONS}

Alopecia areata is a complex illness that is probably due to multiple genetic, autoimmune, and environmental factors; in patients with a genetic predisposition the exposition to one or more environmental factors, although not completely understood, leads to autoimmune reaction against the hair follicles.

The natural history and the treatment response of AA are extremely variable among individuals.

There are several therapeutic strategies available, both topical and systemic. Topical immunotherapy represents a highly effective therapy, mainly in the most challenging clinical forms of $\mathrm{AA}$, and should be the first-choice treatment in adults with AL with scalp extension greater than $50 \%$.

In this study, we evaluated the effectiveness of topical immunotherapy and our data highlight that:

1. Topical immunotherapy has proven to be effective in $44 \%$ of cases.

2. The factors that most affected the outcome of the therapy were:

(a) Severity and extent of AA,

(b) Duration of the disease,

(c) Presence of atopy,

(d) Early development of allergic contact dermatitis.

3. The standard protocol for the application of topical immunotherapy remains the most effective method to have a good response to the therapy while avoiding recurrence.

In the last few years important progress has been made in the treatment of AA, mainly after a clearer definition of etiopathogenetic mechanisms emerged; however, not one of the currently available therapies is curative or efficacious in $100 \%$ of cases or can avoid recurrences.

In the future the goal will be the accurate definition of the pathogenetic mechanisms in order to improve new therapeutic strategies 
allowing an individualized therapeutic approach in the context of personalized medicine.

\section{ACKNOWLEDGEMENTS}

Funding. No funding or sponsorship was received for this study or publication of this article. The article processing charges were funded by the authors.

Authorship. All named authors meet the International Committee of Medical Journal Editors (ICMJE) criteria for authorship for this article, take responsibility for the integrity of the work as a whole, and have given their approval for this version to be published.

Disclosures. Nicola Zerbinati, Cristina Esposito, Edoardo D'Este, Alberto Calligaro, and Rossano Valsecchi have nothing to disclose.

Compliance with Ethics Guidelines. All procedures performed in studies involving human participants were in accordance with the ethical standards of the institutional and/or national research committee and with the 1964 Declaration of Helsinki and its later amendments or comparable ethical standards. Informed consent was obtained from all individual participants included in the study.

Data Availability. All data generated or analyzed during this study are included in this published article.

Open Access. This article is distributed under the terms of the Creative Commons Attribution-NonCommercial 4.0 International License (http://creativecommons.org/licenses/ by-nc/4.0/), which permits any noncommercial use, distribution, and reproduction in any medium, provided you give appropriate credit to the original author(s) and the source, provide a link to the Creative Commons license, and indicate if changes were made.

\section{REFERENCES}

1. Dainichi T, Kabashima K. Alopecia areata: what's new in epidemiology, pathogenesis, diagnosis, and therapeutic options? J Dermatol Sci. 2017;86:3-12.

2. Safavi K. Prevalence of alopecia areata in the first national health and nutrition examination survey. Arch Dermatol. 1992;128:702.

3. Yang S, et al. The genetic epidemiology of alopecia areata in China. Br J Dermatol. 2004;151:16-23.

4. Chu S-Y, et al. Comorbidity profiles among patients with alopecia areata: the importance of onset age, a nationwide population-based study. J Am Acad Dermatol. 2011;65:949-56.

5. Miller R, Conic RZ, Bergfeld W, Mesinkovska NA. Prevalence of comorbid conditions and sun-induced skin cancers in patients with alopecia areata. J Investig Dermatol Symp Proc. 2015;17:61-2.

6. Ruiz-Doblado S, Carrizosa A, García-Hernández MJ. Alopecia areata: psychiatric comorbidity and adjustment to illness. Int $\mathrm{J}$ Dermatol. 2003;42:434-7.

7. Stankler L. Synchronous alopecia areata in two siblings: a possible viral aetiology. Lancet. 1979;1:1303-4.

8. Alsaleh QA, Nanda A, al-Hasawi F, el-Kashlan M. Concurrent appearance of alopecia areata in siblings. Pediatr Dermatol. 1995;12:285-6.

9. Martinez-Mir A, et al. Genomewide scan for linkage reveals evidence of several susceptibility loci for alopecia areata. Am J Hum Genet. 2007;80:316-28.

10. $\mathrm{Lu} \mathrm{W}$, et al. Alopecia areata: pathogenesis and potential for therapy. Expert Rev Mol Med. 2006;8:1-19.

11. Paus R, Nickoloff BJ, Ito T. A 'hairy' privilege. Trends Immunol. 2005;26:32-40.

12. Madani S, Shapiro J. Alopecia areata update. J Am Acad Dermatol. 2000;42:549-66 (quiz 567-570).

13. Messenger AG, McKillop J, Farrant P, McDonagh AJ, Sladden M. British Association of Dermatologists' guidelines for the management of alopecia areata 2012. Br J Dermatol. 2012;166:916-26.

14. Alkhalifah A, Alsantali A, Wang E, McElwee KJ, Shapiro J. Alopecia areata update. J Am Acad Dermatol. 2010;62:177-88.

15. Alkhalifah A, Alsantali A, Wang E, McElwee KJ, Shapiro J. Alopecia areata update: part II. 
Treatment. J Am Acad Dermatol. 2010;62:191-202 (quiz 203-204).

16. DeLeve LD. Dinitrochlorobenzene is genotoxic by sister chromatid exchange in human skin fibroblasts. Mutat Res. 1996;371:105-8.

17. Gulati N, et al. Molecular characterization of human skin response to diphencyprone at peak and resolution phases: therapeutic insights. J Invest Dermatol. 2014;134:2531-40.

18. D'Ovidio R. Alopecia areata: news on diagnosis, pathogenesis and treatment. G Ital Dermatol Venereol. 2014;149:25-45.

19. Ohlmeier MC, Traupe $H$, Luger TA, Böhm $M$. Topical immunotherapy with diphenylcyclopropenone of patients with alopecia areata-a large retrospective study on 142 patients with a selfcontrolled design. J Eur Acad Dermatol Venereol. 2012;26:503-7.

20. Weise K, Kretzschmar L, John SM, Hamm H. Topical immunotherapy in alopecia areata: anamnestic and clinical criteria of prognostic significance. Dermatology (Basel). 1996;192:129-33.
21. Gordon PM, Aldridge RD, McVittie E, Hunter JAA. Topical diphencyprone for alopecia areata: evaluation of 48 cases after 30 months' follow-up. Br J Dermatol. 1996;134:869-71.

22. Firooz A, et al. Topical immunotherapy with diphencyprone in the treatment of extensive and/ or long-lasting alopecia areata. J Eur Acad Dermatol Venereol. 2005;19:393-4.

23. Ajith C, Gupta S, Kanwar AJ. Efficacy and safety of the topical sensitizer squaric acid dibutyl ester in alopecia areata and factors influencing the outcome. J Drugs Dermatol. 2006;5:262-6.

24. van der Steen PH, van Baar HM, Happle R, Boezeman JB, Perret CM. Prognostic factors in the treatment of alopecia areata with diphenylcyclopropenone. J Am Acad Dermatol. 1991;24:227-30.

25. McDonagh AJG, Tazi-Ahnini R. Epidemiology and genetics of alopecia areata. Clin Exp Dermatol. 2002;27:405-9. 